\title{
The Effects of Low Doses of $\Delta-9$ Tetrahydrocannabinol on Reinforcement Processing in the Risky Decision-Making of Young Healthy Adults
}

\author{
Robert D Rogers*,', Judi Wakeley', Phillip J Robson ${ }^{1,2}$, Zubin Bhagwagar' and Petra Makela',2 \\ 'Department of Psychiatry, University of Oxford, Warneford Hospital, Oxford, UK; ${ }^{2}$ GW Pharmaceuticals Ltd, Salisbury, UK
}

\begin{abstract}
Research suggests that risky decision-making is sensitive to neuromodulatory influences acting upon corticolimbic circuitry. However, while other evidence attests to effects of $\Delta-9$ tetrahydrocannabinol (THC) on the activity of reward pathways, relatively little is known about the possible involvement of cannabinoid activity in risky choice. In this experiment, we examined the effects of a single sublingual $5 \mathrm{mg}$ dose of THC on a test of risky decision-making (requiring choices between simultaneously presented gambles differing in their magnitude of gains, magnitude of losses and the probability with which these outcomes were delivered). Tests of non-normative decision-making involving risk-aversion when deciding between gains and risk-seeking choices when deciding between losses were also included. In all, 15 healthy adults were administered $5 \mathrm{mg}$ THC and placebo in a double-blind, placebo-controlled, within-subject, crossover design. THC had three principal effects relative to placebo: (i) THC reduced choice of gambles with variable gains and losses, but increased choice of gambles with zero-expected value; (ii) THC reduced participants' attention towards losses when the probability of winning was low (and the probability of losing was high); and (iii) THC speeded participants' responses to gambles with large compared to small potential gains. These results suggest that THC mediates specific motivational processes and the processing of reinforcement cues during risky choice, perhaps reflecting altered $\mathrm{CB}_{1}$ receptor or catecholamine activity within corticolimbic pathways.

Neuropsychopharmacology (2007) 32, 417-428. doi: 10.1 038/sj.npp. I 30I 175; published online 27 September 2006
\end{abstract}

Keywords: decision-making; $\Delta-9$ tetrahydrocannabinol; cannabis; reward; punishment; $C B_{\text {I }}$ receptors

\section{INTRODUCTION}

Everyday decision-making is often experienced as an emotionally powerful process (Damasio, 1994). Decisionmakers (choosing between occupational or financial opportunities, opportunities to meet partners etc.) are frequently confronted with options associated with a range of motivationally significant but uncertain outcomes that differ in the kind and magnitude of reinforcement. Deciding under such uncertainty requires the capacity to weigh information about potential rewards and punishments, and control the motivational states that they excite, in order to resolve the most effective choice (Rogers et al, 2003). Psychiatric illnesses, such as substance dependent disorders and pathological gambling that involve dysfunctional reinforcement processes, are associated with impaired

\footnotetext{
*Correspondence: Dr RD Rogers, Department of Psychiatry, University of Oxford, Warneford Hospital, Oxford OX3 7JX, UK, Tel: + 44 I865 226 399, Fax: + 44 I865 793 I0।,

E-mail: robert.rogers@psych.ox.ac.uk

Received 3 August 2005; revised 30 May 2006; accepted 7 June 2006 Online publication: 30 June 2006 at http://www.acnp.org/citations/ Npp063006050493/default.pdf
}

decision-making (Bechara et al, 2001; Bechara and Damasio, 2002; Rogers et al, 1999a).

Consistent with the strong emotional component of decision-making, recent pharmacological research indicates that risky choice depends upon the modulatory effects of major neurotransmitter systems. This includes demonstrations that manipulation of serotonergic (Rogers et al, 1999a, 2003), catecholamine (Rogers et al, 2004; Scarna et al, 2005) and GABAergic activity (Deakin et al, 2004; Lane et al, 2005a) can influence laboratory models of decision-making. However, at the current time, little is known about the importance of cannabinoid activity in decision-making despite the preponderance of $\mathrm{CB}_{1}$ receptors within corticolimbic circuitry (Herkenham et al, 1990) and their interactions with other neuromodulatory systems (Ameri, 1999; Tanda et al, 1997).

Several lines of evidence suggest at least a role for cannabinoid activity in decision-making. First, driving studies suggest that, under some circumstances, acute cannabis intake impairs cognitive functions relevant to decision-making cognition (Ramaekers et al, 2004). These include sensori-motor coordination and monitoring functions giving rise to late braking or poor lane control (Hansteen et al, 1976; Liguori et al, 1998; Ramaekers et al, 
2000). By contrast, other studies indicate that individuals under the influence of cannabis are apparently aware that their driving skills are impaired and compensate for this by reducing speed and increasing the distance between themselves and cars ahead, suggesting a reduction in risktaking behavior (Iversen, 2000; Robbe, 1994 for review). In comparison, studies with flying simulations have demonstrated more consistent deficits following cannabis intake (Janowsky et al, 1976). Second, THC can alter choices on operant tasks sustained by reinforcement (Brady and Balster, 1980; Kelly et al, 1993; Kamien et al, 1994; Schulze et al, 1989) while other studies with humans show that THC can produce motivational deficits when working for monetary reward (Foltin et al, 1989; Haney et al, 1997; Miles et al, 1974; Pihl and Sigal, 1978).

Third, THC is known to impact on aspects of reward processing by, for example, reducing reward thresholds for operant responding leading to brain-self-stimulation (Gardner et al, 1988; Gardner and Lowinson, 1991). Also, THC also has an indirect action on dopamine neurotransmission in the mesolimbic and mesocortical systems (Ameri, 1999; Gardner and Vorel, 1998; Tanda and Goldberg, 2003; Tanda et al, 1997), suggesting that the acute effects of THC include altered catecholamine activity within the corticolimbic pathways that mediate decision-making cognition (Scarna et $a l, 2005)$. Other studies in humans indicate that smoked marijuana alters regional cerebral blood flow (ie neural activity) within orbitofrontal and medial prefrontal areas (Mathew et al, 2002; O'Leary et al, 2002; Volkow et al, 1996) known to subserve decision-making function (eg Bechara et al, 1996).

We have developed a method for investigating decision-makers' attention towards (and processing of) reinforcement information while making risky choices. Subjects are asked to make a series of choices between two simultaneously presented gambles, one of which consists of a 0.5 chance to win or lose a certain amount (and has zero expected value) while the other gamble varies in the magnitude of gains (reward), magnitude of losses (punishment), and the probabilities with which these outcomes are delivered (giving rise to expected values that vary between positive and negative values) (Rational choice models have defined rationale/normative decision-making in terms of maximizing an 'expected value' (or derived utility). The expected value of a gamble is the sum of its gains and losses, each weighted by their probability of occurrence (Goldstein and Hogarth, 1997). Variation in subjects' preferences indicated that rapid dietary depletion of the serotonin precursor, 1-tryptophan, altered attention towards information about possible gains, suggesting that serotonin can play a role in the processing of reward information during risky choice (Rogers et al, 2003). By contrast, an acute $80 \mathrm{mg}$ dose of the betaadrenoceptor antagonist, propranolol, diminished attention toward losses when the probability of winning was low (and the probability of losing high), suggesting that noradrenaline modulates processing of punishment cues during stressful choices (Rogers et al, 2004). Finally, a branched-chain amino-acid drink (lacking the catecholamine precursor, tyrosine) reduced attention towards loss and, to a lesser extent, gain information, suggesting that dopamine has a quite general role in the processing of reinforcement cues during risky decision-making (Scarna et al, 2005).

In this study, we investigated the effects of a relatively low dose of THC plant extract (sublingual $5 \mathrm{mg}$ ) on the risky decision-making of healthy adults. In terms of hypothesizing a role for cannabinoid activity in this aspect of cognition, two recently published studies are central. First, Lane and Cherek (2002) examined responding on two concurrently available random interval (5s) schedules delivering monetary reward. One response option delivered a decreasing amount of reward over the course of each study session while the other delivered a constant level of reinforcement. The highest two doses of THC (1.77 and $3.58 \%$ ) induced a tendency to maintain responding on the schedule with a decreasing density of reward, suggesting that cannabinoid activity reduced participants' sensitivity to reinforcement. Against this background, and evidence of motivational changes following marijuana intake (Foltin et al, 1989; Kagel et al, 1980; Pihl and Sigal, 1978), one might hypothesize that sublingual THC will reduce discrimination between response options with zero and those with varying expected value.

Second, Lane et al (2005b) examined responding on two concurrently available variable ratio schedules, again delivering monetary reward. One option (labeled the 'nonrisky' option) delivered a small certain gain with a probability of 1.0 (expected value $=\$ 1.12)$; the other option (labeled the 'risky' option) delivered larger gains and losses with a probability of 0.5 (expected value $=\$ 0$ ). The highest dose of THC (3.58\%) increased choice of the risky option and induced greater persistence in such selections regardless of the outcomes of previous decisions. Against this background, and evidence that THC enhances rewardrelated processing (Gardner et al, 1988), one might hypothesize that sublingual THC increases participants' attention towards reinforcement cues when they make risky choices.

\section{MATERIALS AND METHODS}

\section{Subjects}

Seven healthy males and eight healthy females participated. General Practitioner approval was obtained, and a full medical history and examination were undertaken prior to testing. Participants were screened for physical illness, and for clinically significant psychiatric illness using the Structured Clinical Interview for DSM-IV Axis I Disorders (First et al, 1996). Six participants reported less than 10 lifetime uses of cannabis; four reported between 10 and 100; and five reported more than 100 . The study was approved by the Oxford Psychiatric Research Ethics Committee and written informed consent was given by all participants. Their mean age was $24.3 \pm 1.3$ years (SE) (19-36 years), and their mean verbal IQ, as estimated by the National Adult Reading Test (Nelson, 1982), was 115.0 \pm 1.3 (106-123).

\section{Design}

This study consisted of a double-blind, placebo-controlled, within-subject, crossover design. Eight participants received 
placebo on the first study day and $5 \mathrm{mg} \Delta$-9-tetrahydrocannabinol (THC) on the second day; seven received THC on the first day and placebo on the second day. Study days were separated by at least 1 week. Participants were asked to abstain from alcohol intake for $24 \mathrm{~h}$ and caffeine for $12 \mathrm{~h}$ prior to each study day. On attendance at the start of each study day, urine was screened for amphetamines (A), benzodiazepines, cocaine, methamphetamine, opiates and THC using immunometric assay kits. Breath alcohol was also tested. A pregnancy test was performed for female participants.

\section{Materials}

THC extracted from standardized plants was administered in an ethanol and propylene glycol solution at $8.5 \mathrm{mg} / \mathrm{ml}$ concentration, using a pump-action sublingual spray. The pharmacokinetics of this method of THC administration indicates relatively wide inter-subject variability in terms of AUC values; however, a Phase I trial with healthy adults suggests that mean plasma concentration values for THC tends to peak between 2 and $3 \mathrm{~h}$ postdosing (Guy and Robson, 2003). The total dose delivered was $5 \mathrm{mg}$ THC in six actuations at $6 \mathrm{~min}$ intervals, with blood pressure and heart rate monitoring $5 \mathrm{~min}$ after each actuation. Dosing was incremental to allow termination in the event of significant adverse events. The matching placebo employed a peppermint flavoring to aid blinding.

Addiction research center inventory (Haertzen and Hickey, 1987). The Addiction research center inventory (ARCI) is a 53-item instrument consisting of six empirically derived subscales that are sensitive to various classes of drug effects: the Morphine-Benzedrine subscale (MBG) is a measure of euphoria; the Pentobarbital-ChlorpromazineAlcohol (PCAG) is a measure of sedation; the $A$ and Benzedrine-Group (BG) are measures of stimulant-like effects; the Lysergic acid (LSD) subscale is a measure of dysphoria and somatic effects; and the Marijuana (M) subscale is sensitive to marijuana effects.

Visual analog scales (Bond and Lader, 1974). Subjective effects were assessed using 16 visual analogue scales (VAS) that provide subscales to measure the following: mental sedation (M), physical sedation (P), feelings of tranquility $(\mathrm{T})$, and other sociability feelings $(\mathrm{S})$.

\section{Decision-Making Task}

On each trial, participants were asked to choose between two simultaneously presented gambles. Each gamble was represented visually by a histogram, the height of which indicated the relative probability of gaining a given number of points (see Figure 1a). The possible gains were indicated in green ink above the histogram; the possible losses were indicated in red ink underneath the histogram. One gamble (colored yellow) was the control gamble, consisting of a 0.50 probability of winning 10 points and a 0.50 probability of losing 10 points (yielding an expected value of 0 ). The alternative 'experimental' gamble (colored blue) varied in the probability of winning which was either high or low (0.75 vs 0.25$)$, possible gains which were large or small ( 80
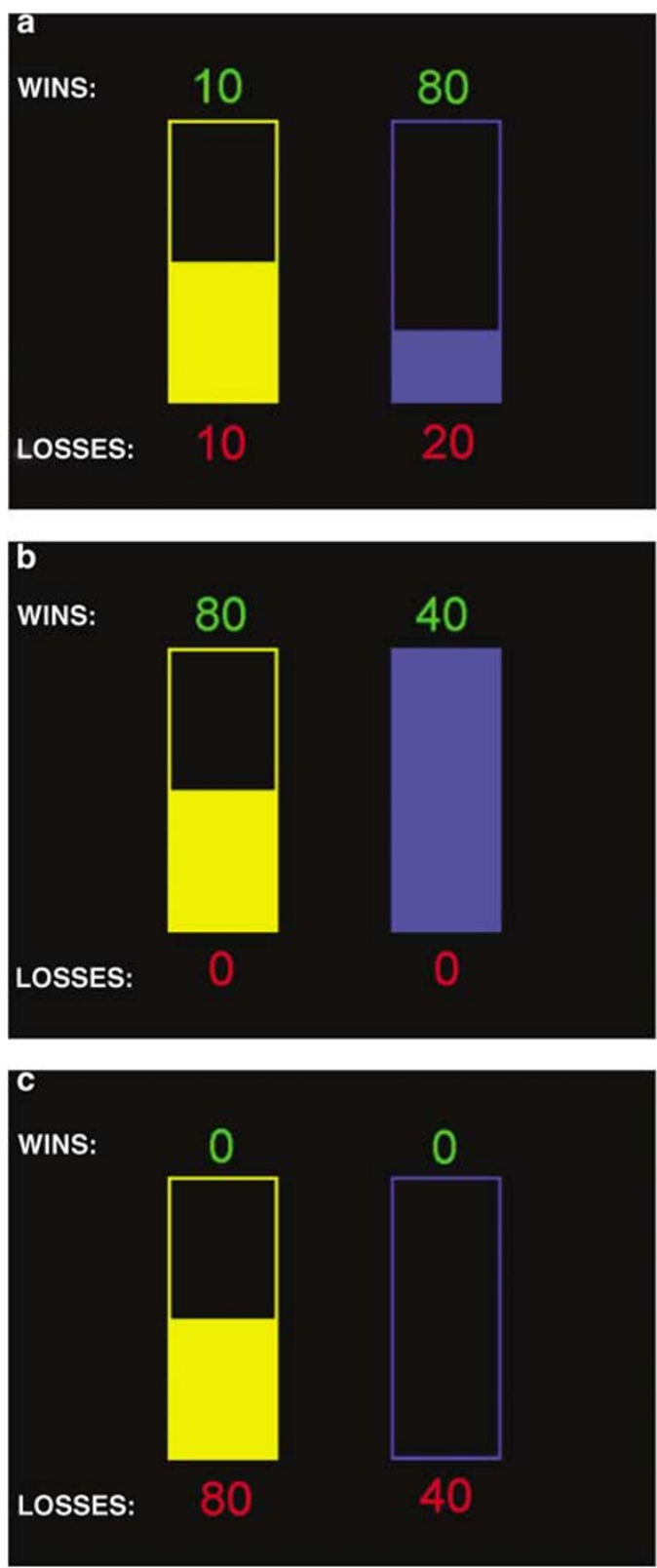

Figure I One example visual display from the risky choice task (Rogers et al, 2003) consisting of an 'experimental' gamble with a 0.25 chance of winning 80 points and a 0.75 chance of losing 20 points vs the control gamble with a 0.50 chance of winning 10 points and a 0.50 of losing 10 points (a). A 'Gains only' trial from the decision-making task consisting of a certain win of 40 points vs 0.50 chance of winning 80 points or 0 points (b). A 'Losses only' trial consisting of a certain loss of 40 points vs a 0.50 chance of a loss of 80 points or 0 points (c).

vs 20 points), and possible losses which were either large or small (80 vs 20 points). These variables were completely crossed to produce eight trial types or gambles with expected values that varied between -55 and +55 (see Table 1). Figure 1a shows an 'experimental' gamble with a 0.25 chance of winning 80 points (and a 0.75 chance of losing 20 points).

The control and 'experimental' gambles appeared randomly on the left or right of the display. Participant were required to press the ' 1 ' or ' 2 ' key on the computer keyboard to indicate choice of the gamble presented on the 
Table I The Eight Types of 'Experimental' Gamble Resulting from the Combination, in a Completely Crossed Design, of Two Levels of Probability, Possible Gains and Possible Losses

\begin{tabular}{lccc}
\hline Probability & $\begin{array}{c}\text { Possible } \\
\text { gains }\end{array}$ & $\begin{array}{c}\text { Possible } \\
\text { losses }\end{array}$ & $\begin{array}{c}\text { Expected value } \\
\text { (points) }\end{array}$ \\
\hline High (0.75) & Large (80) & Large (80) & 40 \\
& & Small (20) & 55 \\
& Small (20) & Large (80) & -5 \\
& & Small (20) & 10 \\
Low (0.25) & & -40 \\
& Large (80) & Large (80) & 5 \\
& Small (20) & -55 \\
& Small (20) & Large (80) & -10 \\
\hline & & Small (20) & \\
\hline
\end{tabular}

The 'expected value' for each gamble equals the sum of its gains and losses, each weighted by their probability of occurrence (Goldstein and Hogarth, 1997). These vary between -55 and +55 points, with a mean of 0 .

left or right. Dependent measures were the proportion of choices of the 'experimental' over control gamble as a function of its probability of winning, size of possible gains and size of possible losses ('proportionate choice'), and the mean deliberation time (ms) for these choices.

As previously described (Rogers et al, 2003), we also included two extra trial types that involve the nonnormative biases of risk-aversion and risk-seeking choices when confronted with certain wins or certain losses (the 'reflection effect'; see Kahneman and Tversky, 1979). The first such trial type was a 'gains only' trial in which the participants were presented simultaneously with a guaranteed win of 40 points $v s$ a 0.5 chance of winning 80 points and a 0.5 chance of losing 0 points (see Figure $1 \mathrm{~b}$ ). Neither option involved any associated losses. By contrast, in the 'losses only' trial type, the participants were presented simultaneously with a guaranteed loss of 40 points $v s$ a 0.5 chance of losing 80 points and a 0.5 chance of losing 0 points (see Figure 1c). Neither option offered any associated gains. Within both the 'gains only' and 'losses only' trials, the expected value of each gamble was equal; however, decision-makers usually exhibit a marked risk-aversion in the former case (ie choice of the guaranteed gain of 40 points) but marked risk-seeking behavior in the latter case (ie choice of the gamble with a 0.5 chance of losing 80 points and a 0.5 chance of losing 0 points) (Schneider and Lopes, 1986). The dependent measures for the 'gains only' trials and the 'losses only' trials were the proportion of trials on which the participants chose the guaranteed outcome and the associated mean deliberation time (ms) for these choices.

All 10 trial types were presented pseudo-randomly within four blocks of 20 trials. At the beginning of each block, participants were given 100 experimenter-defined points, and asked to make choices which would increase this amount by as much as possible. These points had no monetary value. Visual feedback was given on the outcome of each choice, and the revised points total was then presented for $2 \mathrm{~s}$ before the next trial. Across the four blocks, there were eight repetitions of each 'experimental' gamble and eight repetitions of each of the 'gains only' and the 'losses only' trial types.

\section{Procedure}

Participants attended the laboratory at 0830 in the morning, and completed the VAS and ARCI prior to dosing, at $+2 \mathrm{~h}$ postdosing (just before administration of the cognitive test battery), +4 and $+6 \mathrm{~h}$ postdosing. The dosing was followed by $90 \mathrm{~min}$ during which participants were supervised and asked not to sleep. At $+2 \mathrm{~h}$, the participants completed the decision-making task alongside other cognitive tasks not described here. Participants were assessed by a doctor before discharge.

\section{Statistics}

The data were analyzed using the Statistical Package for Social Scientists version 12.0 (SPSS). Ratings data from the VAS and ARCI subscales were analyzed with multifactorial repeated measures analyses of variance (ANOVAs) with the two between-subject factors of gender and treatment order (placebo/THC vs THC/placebo) and the two within-subject factors of treatment (placebo $v s$ THC) and time (baseline $v s$ $+2,+4$, and $+4 \mathrm{~h}$ ). For the risky decision-making task, the proportionate choice data were arcsine-transformed, as is appropriate whenever the variance of a measure is proportional to its mean (Howell, 1987); however, all of the data reported in the text, figures and tables describe untransformed values. These data, and the mean deliberation times, were analyzed with repeated measures ANOVAs with the two between-subject factors of gender and order of treatment and the four within-subject factors of treatment, probability of winning (high $v s$ low), size of possible gains (large $v s$ small) and size of possible losses (large $v s$ small). The 'gains only' and 'losses only' trials were analyzed with repeated measures ANOVAs with the two between-subject factors of gender and treatment order, and the two withinsubject factors of treatment and trial type ('gains only' $v s$ 'losses only').

The importance of subjective changes in mediating the treatment-related effects on risky decision-making were assessed by repeating the above analyses with the inclusion of ARCI and VAS subscale ratings as covariates (calculated as the change in ratings following THC against placebo treatment). There is evidence that the extent of previous use of cannabis can influence its effects in different individuals (Kirk and de Wit, 1999). Therefore, we also repeated the above analyses with the inclusion of an extra betweensubject factor of previous experience - minimal (less than 10 lifetime uses), moderate (between 10 and 100); and frequent (more than 100 uses) - to investigate any such effects.

\section{RESULTS}

All participants tested negative for alcohol and other illicit drugs on both study days, and were able to tolerate six dose increments (as described above), to receive the full dose of $5 \mathrm{mg}$ THC. 


\section{Subjective Effects}

Addiction research center inventory. THC significantly increased self-reported marijuana effects compared to placebo $\quad(1.63 \pm 0.40 \quad v s \quad 0.33 \pm 0.16) \quad(\mathrm{F}(1,11)=14.64$, $p<0.005)$, with a trend for this effect to be maximal from $+2 \mathrm{~h}$ onward $(\mathrm{F}(3,33)=5.17, p=0.05)$ (see Figure 2a; M-subscale). THC treatment also significantly increased sedative effects compared to placebo treatment $(5.55 \pm 0.82$ vs $4.28 \pm 0.74)(\mathrm{F}(1,11)=6.42, p<0.05)$ (Figure 2b; PCAGsubscale). By contrast, THC did not produce any significant increase in euphoria in comparison with placebo $(\mathrm{F}(1,11)=1.85)$ (Figure 2c; MBG-subscale); neither did it produce marked amphetamine-like effects $(\mathrm{F}(1,11)=1.07)$ (Figure 2d; A-subscale). Stimulant effects associated with the benzedrine family of drugs were reduced following THC compared to placebo treatment although not significantly so $(\mathrm{F}(3,33)=1.79) \quad$ (Figure 2e; BG-subscale). Finally, dysphoria and somatic effects tended to increase after dosing $(\mathrm{F}(1,11)=2.70, p=0.06)$ (Figure 2f; LSD subscale); however, this increase was not significantly greater following THC compared to placebo treatment $(F<1.00)$.

Visual analog scales. THC treatment was associated with significantly higher ratings of mental sedation compared to placebo treatment $(179.69 \pm 17.25$ vs $139.74 \pm 17.71)$ $(\mathrm{F}(1,10)=8.75, p<0.05) \quad($ Data for the mental sedation subscale of the VAS were unavailable for one participant.), increasing significantly over $+6 \mathrm{~h}$ following dosing with THC while remaining unchanged following treatment with placebo $(\mathrm{F}(3,30)=5.83, p<0.05)$ (see Figure 3a). Additionally, THC increased physical sedation compared to placebo $(163.60 \pm 13.41$ vs $134.02 \pm 10.86) \quad(\mathrm{F}(1,9)=9.72$, $p<0.05)$. This THC-induced increase was maximal at $+4 \mathrm{~h}$ postdosing $(F(3,27)=7.63, p<0.005)$ (Figure 3b). Feelings of tranquillity did not differ following THC compared to placebo treatment $(111.05 \pm 9.95$ vs $113.90 \pm 9.63)$ $(\mathrm{F}(1,10)<1.0)$ (Figure 2c). Finally, feelings of sociability were reduced over the course of the study day following placebo treatment but increased following THC treatment $(\mathrm{F}(3,30)=4.63, p<0.01)$ (Figure $3 \mathrm{~d})$.

\section{Decision-Making Task}

Proportionate choice. All participants chose the 'experimental' gamble significantly more often when its probability of winning was high compared to when it was low $(0.79 \pm 0.03$ vs $0.19 \pm 0.03) \quad(\mathrm{F}(1,11)=130.16, p<0.001)$ (see Figure 4a). Similarly, all participants chose the 'experimental' gamble significantly more often when its possible gains were large compared to when its possible gains were small $(0.60 \pm 0.02$ vs $0.38 \pm 0.03)(\mathrm{F}(1,11)=$ $38.44, p<0.001$ ) (see Figure $4 \mathrm{~b}$ ), but significantly less often when its losses were large compared to when they were small $(0.37 \pm 0.03$ vs $0.61 \pm 0.02)(\mathrm{F}(1,11)=28.85, p<0.001)$ (Figure 4c). While these patterns of choice were not generally altered following THC compared to placebo (all Fs $<1.00$ ), THC treatment did produce a highly significant reduction in choice of the 'experimental' gamble compared to placebo $(0.46 \pm 0.01$ vs $0.52 \pm 0.02) \quad(\mathrm{F}(1,11)=22.95$, $p<0.001$ ) (see Figure $4 \mathrm{a}-\mathrm{c}$ ).

Additionally, the reduction in participants' choice of the 'experimental' gamble when its associated losses were large compared to when they were small was not altered after THC compared to placebo treatment when the probability of winning was high (see Figure 5a), but was significantly diminished when the probability of winning was low (see
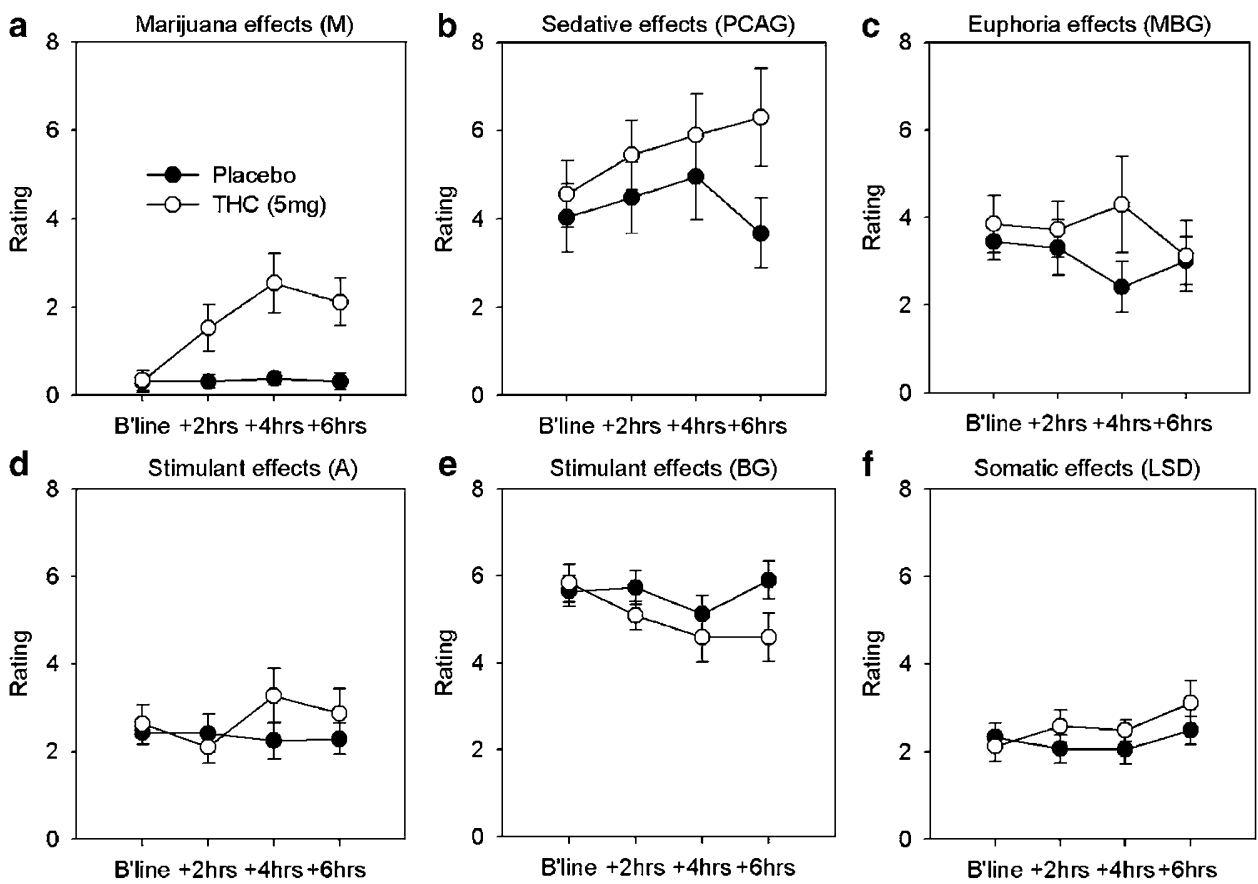

Figure 2 Mean self-report ratings ( \pm SE) on subscales of the ARCI (Haertzen and Hickey, 1987) following treatment with $5 \mathrm{mg}$ sublingual THC and treatment with placebo. Marijuana subscale (M) (a). PCAG subscale (b). MBG (c). Amphetamine subscale (A) (d). Benzedrine-group (BG) subscale (e). and Lysergic acid (LSD) subscale (f). 

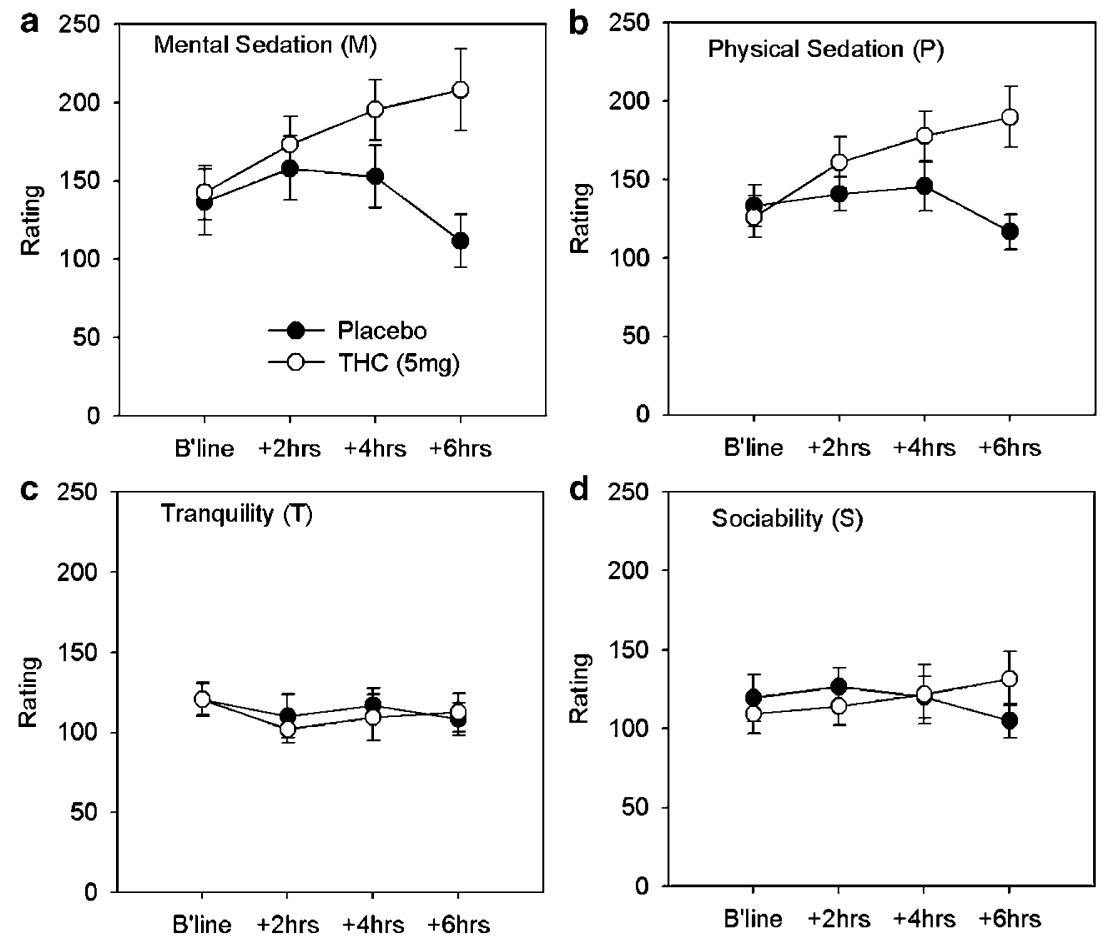

Figure 3 Mean ratings ( \pm SE) on Visual Analog Scales for drug effects (VAS; Bond and Lader, 1974) following 5 mg sublingual THC and placebo. Mental sedation (M) (a). Physical sedation (P) (b). Feelings of tranquillity (T) (c) and feelings of sociability (S) (d).
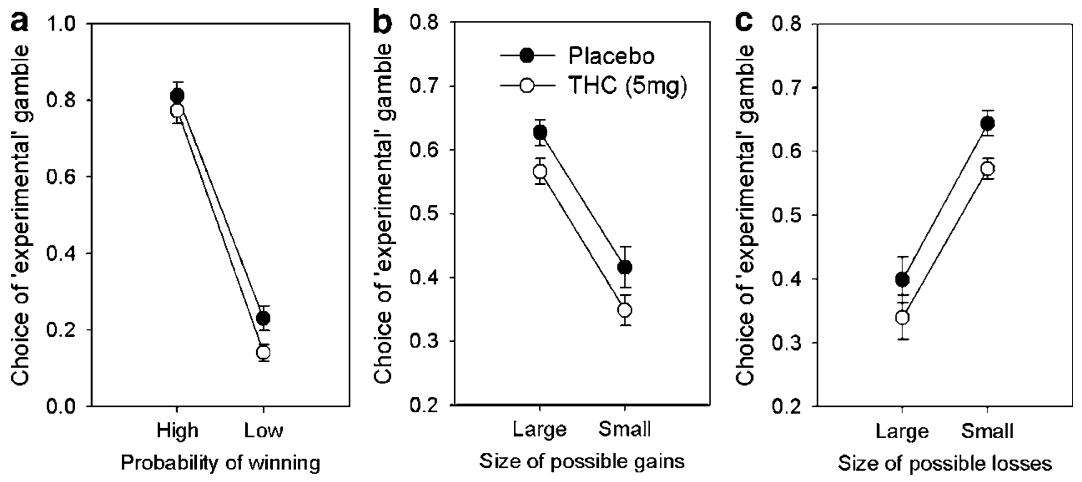

Figure 4 Proportion of choices of the 'experimental' over the control gamble following administration of $5 \mathrm{mg}$ sublingual THC and placebo for the three manipulated factors. High vs low probabilities of winning on the 'experimental' gamble (a). Large vs small possible gains (b). Large vs small possible losses (c). Main effect of treatment (placebo vs THC): $F(I, I I)=22.95, p<0.000 I$.

Figure $5 b$ ), as evidenced by a highly significant 3-way interaction between treatment, probability of winning and size of possible losses $(F(1,11)=6.59, p<0.05)$. Analyses of the simple interaction effects confirmed that THC treatment reduced participants' choice of the 'experimental' gamble in response to larger possible losses to the same extent as placebo when its associated probability of winning was high $(\mathrm{F}(1,11)=2.08)$ but not when its probability of winning was low (see Figure $5 \mathrm{~b})(\mathrm{F}(1,11)=9.71, p=0.01)$.

By contrast, there was no indication that the increased choice of the 'experimental' gamble when its potential gains were large compared to when they were small was significantly changed following the THC treatment compared to the placebo treatment as a function of the probability of winning (or losing) on the 'experimental' gamble $(\mathrm{F}(1,11)=1.66)$ (see Figure $5 \mathrm{c}$ and $\mathrm{d}$ ).

Overall, there was no difference in the extent to which male and female participants chose the 'experimental' gamble during performance of the risky choice task $(0.49 \pm 0.02$ vs $0.49 \pm 0.02)(F<1.00)$. There were no other significant interactions involving gender, treatment, treatment order, or any other task variable. Repeat analyses, including subjective effects (calculated as change following THC compared to placebo treatment) as covariates did not alter the pattern of results reported above. However, entering age and estimated verbal IQ revealed that both variables acted as significant or near-significant covariates $(\mathrm{F}(1,9)=4.72, p=0.06$ and $\mathrm{F}(1,9)=6.7, p<0.05$, respec- 

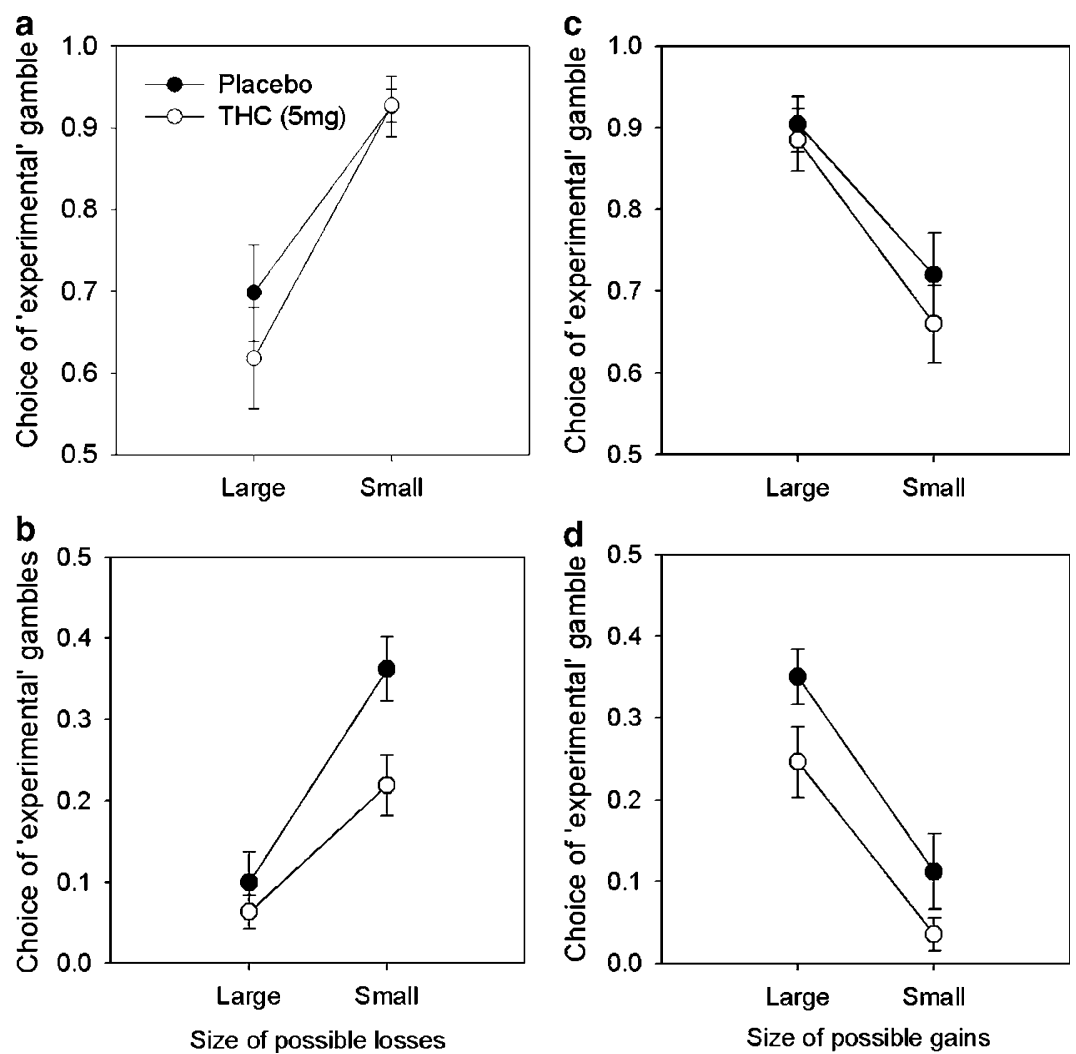

Figure 5 Proportion of choices of the 'experimental' gamble over the control gamble following treatment with $5 \mathrm{mg}$ sublingual THC and treatment with placebo. Large vs small possible losses when the probability of winning was high (a). Large vs small possible losses when the probability of winning was low (b). Large vs small possible gains when the probability of winning was high (c). Large vs small possible gains when the probability of winning was low (d). Treatment (placebo vs THC) $\times$ probability of winning (high vs low) $\times$ size of possible losses (large vs small) $(c$ and $d): F(I, I I)=6.59, p<0.05$; simple interaction effect when probability of winning was low (d): treatment $\times$ size of possible losses: $F(I, I I)=9.7 I, p=0.01$.

tively) which abolished the statistical significance of the reduced choice of the 'experimental' gamble following THC treatment $(F<1.00)$. The inclusion of these covariates also abolished the statistical significance of the reduced discrimination between the size of possible losses when the probability of winning on the 'experimental' gamble was low following THC compared to placebo treatment $(F<1.00)$. There was no indication that extent of previous cannabis use significantly influenced participants' risky choices.

Deliberation times. Participants were significantly faster to make their choices when the probability of winning on the 'experimental' gamble was high compared to when it was low $(1769 \pm 161$ vs $1994 \pm 191 \mathrm{~ms})(\mathrm{F}(1,11)=15.46$, $p<0.005)$. This facilitatory effect was no greater following THC compared to placebo treatment (see Table 2) $(F<1.00)$. By contrast, while participants made slightly slower choices when the 'experimental' gamble was associated with larger possible gains compared to smaller possible gains following placebo treatment, they made markedly faster responses following THC treatment (see Table 2) $(\mathrm{F}(1,11)=4.91, p<0.05)$. Participants also tended to make slower decisions when the 'experimental' gamble was associated with large possible losses compared to small possible losses $(1953 \pm 178$ vs $1810 \pm 178 \mathrm{~ms})$ $(\mathrm{F}(1,28)=3.67, p=0.09)$; however, there was no difference in this inhibitory effect following THC and placebo treatments (see Table 2) $(F<1.00)$.

Overall, THC treatment was associated with only slightly faster decisions than placebo treatment $(1833 \pm 184$ vs $1929 \pm 202 \mathrm{~ms}$ ) while female participants made slightly faster decisions than male participants $(1758 \pm 237 v s$ $2005 \pm 256 \mathrm{~ms})$. Neither of these differences were reliable $(F<1.00)$. There were no other significant interactions involving gender, treatment, or treatment order. Entering subjective effects as covariates did not alter the above pattern of statistics. Entering age and estimated verbal IQ as covariates revealed that neither variable were significant covariates $(F \mathbf{s}<1.00)$, but that the two way interaction by which placebo treatment slowed choices when the 'experimental' gamble was associated with larger possible gains compared to smaller possible gains, while THC treatment speeded these choices, was rendered nonsignificant. Finally, there was no evidence that extent of previous cannabis use significantly influenced participants' performance of the risky choice task.

\section{'Gains Only' vs 'Losses Only' Trials}

Proportionate choice. Participants chose the guaranteed outcome significantly more often on the 'gains only' trials (offering a choice between a certain gain of 40 points and a 0.50 chance of 80 points or 0 points) than on the losses 
Table 2 Mean Deliberation Times (ms) Following Treatment with $5 \mathrm{mg}$ THC and Placebo for Trials on Which the 'Experimental' Gamble was Associated with High vs Small Probability of Winning, Large vs Small Possible Gains, and Large vs Small Possible Losses

\begin{tabular}{|c|c|c|c|c|c|c|}
\hline & \multicolumn{2}{|c|}{ Probability of winning } & \multicolumn{2}{|c|}{ Possible gains } & \multicolumn{2}{|c|}{ Possible losses } \\
\hline & High & Low & Large & Small & Large & Small \\
\hline THC (5 mg) & $1734 \pm 161$ & $1932 \pm 211$ & $1714 \pm 155$ & $1953 \pm 220$ & $1907 \pm 202$ & $1760 \pm 170$ \\
\hline
\end{tabular}
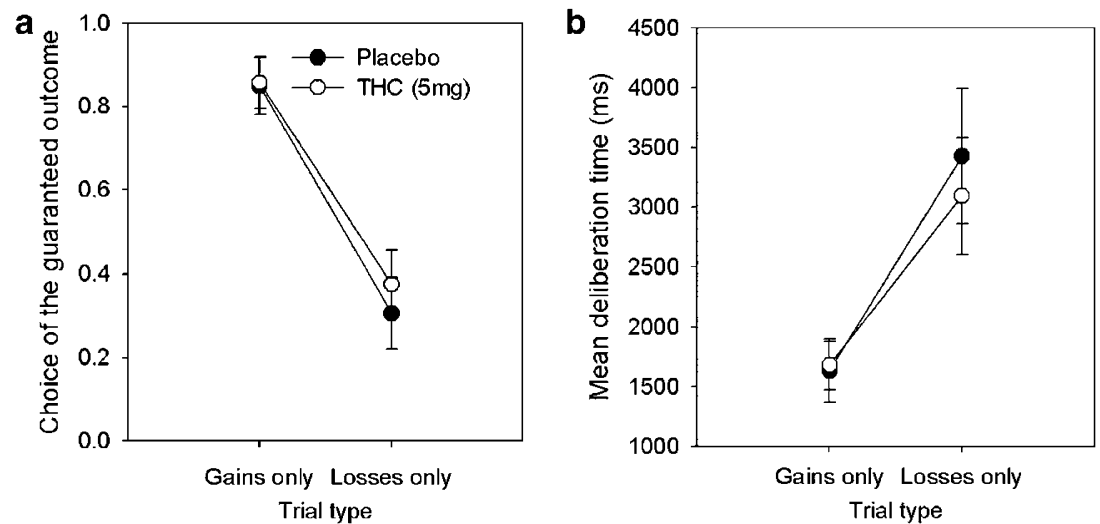

Figure 6 Proportion of choices of the guaranteed outcome following treatment with $5 \mathrm{mg}$ sublingual THC and treatment with placebo on the 'gains only' trials (offering a choice between a certain gain of 40 points and a 0.50 chance of 80 points or 0 points) and the 'losses only' trials (offering a certain loss of 40 points or a 0.50 chance of a loss of 80 points or 0 points) (a). Mean deliberation times (ms) for choices following THC and placebo treatment on the 'Gains only' and 'Losses only' trials (b).

only' trials (offering a certain loss of 40 points or a 0.50 chance of a loss of 80 points or 0 points $)(F(1,11)=24.32$, $p<0.001$ ). However, this pattern of choices (risk-aversion when choosing between gains and risk-seeking when choosing between losses) was not significantly different following the THC compared to placebo treatments $(\mathrm{F}(1,11)=1.14) \quad$ (see Figure 6a). There were no other significant interactions involving gender, treatment, or treatment order and no suggestion that age or estimated IQ were significant covariates that might have influenced the above findings.

Deliberation times. Participants were significantly quicker to make their choices on the 'gains only' trials compared to the 'losses only' trials $(1654 \pm 215$ vs $3258 \pm 492 \mathrm{~ms})$ $(\mathrm{F}(1,11)=10.65, p<0.001)$, with no difference in the size of this effect following THC treatment $(1678 \pm 203$ vs $3089 \pm 489 \mathrm{~ms})$ compared to placebo treatment $(1630 \pm 266$ vs $3426 \pm 565 \mathrm{~ms}) \quad(F<1.00)$ (see Figure $6 \mathrm{~b})$. Gender, treatment, or treatment order did not influence these effects; and there was no evidence that either age or estimated IQ act as significant covariates in the above analyses.

\section{DISCUSSION}

The present study demonstrates that administration of low doses of THC had relatively complex effects on the risky decision-making of healthy adults. Specifically, sublingual
THC reduced choice of gambles associated with highly variant expected value, but increased choice of gambles with zero-expected value. THC also reduced attention towards (and processing of) prospective losses when the probability of winning was low (and the probability of losing was high). Finally, THC speeded decisions about gambles with large gains compared to small gains. Before considering the significance of these results, we consider some methodological issues that are relevant to interpretation.

First, sublingual doses of THC produced subjective effects of cannabis administration that were broadly similar to those reported in an earlier study involving the same dose administered by the same means (Makela et al, 2006) and previously published reports of the subjective effects of oral doses of Marinol (Kirk et al, 1998; Kirk and de Wit, 1999). Specifically, THC induced statistically significant increases in marijuana-like effects as measured by the $M$ subscale of the ARCI (Haertzen and Hickey, 1987). Subjective effects associated with THC intake did not include marked changes in participants' euphoria (BG subscale of the ARCI); nor were there were significant changes in stimulant-like effects (A and MBG subscales). The single difference between the present findings and those of Makela et al (2006) and Kirk et al (1998) is that dysphoria and somatic symptoms (LSD subscale) were not differentially increased following treatment with THC compared to treatment with placebo.

As expected, THC was associated with mild increases in both physical and mental sedation as measured by the VAS (Bond and Lader, 1974) and the PCAG subscale of the ARCI (Haertzen and Hickey, 1987), making it at least possible that 
the cognitive effects of the THC treatment identified in the present study reflect (in part) nonspecific changes in alertness. However, it is unlikely that the effects of THC on risky decision-making can be attributed solely to such effects. This is because, in a separate study with the same dose of sublingual THC, we observed similar sedative effects accompanied by improved performance on a measure of short-term spatial working memory (Makela et al, 2006). Additionally, in the present study, participants' deliberation times for their risky choices were not uniformly increased following THC treatment in a way suggestive of a generalized motivational deficit.

Second, there are, of course, many limitations to laboratory models of human behavior including, in this case, the rather abstract (and perhaps overly mathematical) nature of choices presented to ours participants. In particular, these choices involved only 'points' reward that had no monetary value. Consequently, our results may, in part, reflect a reduction in motivation associated with the use of such notional reinforcers. Without a direct comparison between the effects of THC on choices involving monetary and nonmonetary reward, this issue must remain unresolved. However, studies of the cognitive psychology of human decision-making have consistently required volunteers to make choices between a variety of notional reinforcers (with no real monetary value), yet have provided valid models of real-life decision-making between real goods and services, and to be effective methods for testing between competing theories of choice under uncertainty (von Winterfeldt and Edwards, 1986; Kahneman and Tversky, 2000). Similarly, the now large body of work on the neuropsychology of decision-making completed with the Iowa gambling task (Bechara et al, 1996) and other tasks (Rahman et al, 2001) has, for the most part, involved facsimile money or points rewards but still provided helpful insights into the decision-making impairments exhibited by clinical populations (Bechara et al, 2002). Risky choices between notional reinforcers have also been shown to be associated with significant activity within reinforcement circuitry (Rogers et al, 1999b), suggesting that such choices recruit neural circuitries involved in the processing of incentive-motivational information (Dayan and Balleine, 2002) and which are subject to the modulatory effects of neurotransmitters, including the cannabinoid system.

Notably, entering changes in subjective state as covariates made no difference to the statistical significance of the THC-induced changes in risky choice. By contrast, age, and estimated IQ were significant covariates of task performance, and their inclusion in the statistical analysis markedly reduced the reliability of THC-related changes in decision-making. Accumulating research suggests that pharmacological effects upon cognitive performance depend upon a number of factors including levels of motivation and baseline cognitive abilities (Miller et al, 1977; Luciana et al, 1992; Gevins et al, 2002; Mehta et al, 2004). Several studies suggest that cognitive effects tend to be greatest in participants with relatively poor baseline (or placebo-related) performance. The present results indicate that THC administration interacted with a comparatively broad measure of cognitive ability - an estimate of verbal IQ - and that removing this source of variance reduces the impact of THC treatment on participants' risky choice. The significance of this finding is unclear. However, one possibility is that participants with higher verbal IQ scores paid closer attention to the different reinforcement signals when contained in the choice displays and, consequently, were less sensitive to the effects of THC treatment. Further research will be needed to clarify this issue.

As noted above, risky decision-making involves the integrated processing of information about candidate actions associated with uncertain gains and losses. Processing information about potential gains (ie rewards) will excite an appetitive/approach motivational stance (towards a given response option) while processing information about potential losses (ie punishments) will excite an aversive/withdrawal motivational stance. Effective choice depends upon balancing these motivational states in order to select a response most consist with current behavioral objectives. Two recent studies of the effects of THC on choice behavior, using concurrent (random and variable ratio) operant schedules suggest that THC may induce risktaking behavior by altering sensitivity to reinforcement and promoting a tendency to continue choosing risky options (Lane and Cherek, 2002; Lane et al, 2005b). The present results add to this picture by providing at least some support for these suggestions and by demonstrating that low doses of THC (sublingually administered) can produce relatively complex effects that might, in different ways, inhibit or promote risky behavior.

First, these data show that sublingual doses of THC reduced participants' choice of gambles with varying gains and losses but increased their choice of gambles with a zeroexpected value (see Figures 4). Such a shift in preference away from gambles with high variant outcomes towards gambles with low variant outcomes is consistent with previous reports that cannabis intake can be associated with reduced risky behavior whereby treated participants adopt cautious cognitive strategies to compensate for ill-effects of drug intake (Iversen, 2000; Robbe, 1994). This aspect of THC's effects on risky choice may also reflect a specific motivational change involving reduced discrimination between those response options with varying expected values (and resultant reinforcement) and those with minimal or zero expected value in the context of instrumental learning (Lane and Cherek, 2002).

Second, the present results indicate that, not withstanding its effects on participants' preferences for high-variant over low-variant gambles, THC also altered attention towards certain reinforcement cues (eg potential losses) but under relatively restricted circumstances (eg when the probability of winning was low and the probability of losing was high; see Figure 5). As noted above, effective decision-making under uncertainty depends upon integrating information about the good and bad consequences of the available response options. Therefore, a failure to attend adequately towards potential losses when the probability of a good outcome is relatively low (and the probability of a bad outcome is relatively high) might constitute a mechanism that promotes the selection of maladaptive responses in difficult choices such as, for example, when attention needs to be allocated to danger signals under conditions of particularly high risk (Robbe, 1994). 
Previous studies also suggest that altered choices following THC administration reflects changed processing of their consequences (Lane et al, 2005b); however, our data indicate that THC can also affect processing of reinforcement information while individuals deliberate between risky options. The subsidiary finding that THC administration speeded responses to gambles with large compared to small gains, while placebo retarded such responses, provides some limited confirmation of the prediction-based upon evidence that $\mathrm{CB}_{1}$ activation enhances reward-related processing (Gardner et al, 1988; Gardner and Lowinson, 1991) - that THC intake will potentiate attention towards appetitive cues during risky choice.

Third, several theorists have postulated that decisionmaking in the face of incomplete information (eg about the probabilities of different outcomes) may involve different cognitive processes to decision-making in the face of more complete information (Baron, 2000 for review). Studies with operant schedules have suggested that THC affects decisionmaking in the former situation (Lane et al, 2005b); the present results suggest that these effects of THC may generalize to situations where the choices are quite precisely specified, perhaps involving other cognitive sequelae of THC intake. Finally, operant studies of choice behavior following THC administration have also noted that response rates are inevitably reduced alongside any changes in the selection of certain choices over others. While it is unlikely that diminished responding can account for the effects of THC in these experiments (see Lane and Cherek, 2002 for discussion), the use of a discrete-trial choice paradigm in the present study provides additional (and reassuring) evidence that the complex effects of THC on risky decisionmaking in human adults are not simple artifacts of diminished response rates (cf. Curran et al, 2002).

We have previously used the current task paradigm to examine the effects of manipulations of the monoaminergic systems on risky decision-making. These studies have consistently demonstrated changes in participants' attentional processing of potential gains and losses (Rogers et al, 2003, 2004; Scarna et al, 2005) rather than reducing participants' discrimination between gambles with varying expected value and gambles with zero expected value as seen in the present experiment. This result is reminiscent of the finding that THC sustains participants' choice of response options associated with ever-diminishing reward (Lane and Cherek, 2002) and suggests that cannabinoid activation may play a complementary role to that of the major monoamine systems by mediating sensitivity to altered reinforcement contingencies associated with response options. Our finding that THC reduced attention towards prospective losses when the probability of winning on the 'experimental' gamble was low and the probability of losing high matches that found in a previous study with an acute dose of $80 \mathrm{mg}$ of the beta-adrenoceptor antagonist, propranolol (Rogers et al, 2004). The mildly sedative action of both these drugs may have the primary/immediate effect of reducing attentional processing of punishment cues while performing a predominantly appetitively motivated task, and thereby promoting risky choice.

An extensive literature details how, in many situations, human decision-makers make highly non-normative decisions (eg Kahneman and Tversky, 1979). In this study, we tested this aspect of decision-making using the 'gains only' and 'losses only' trials in which participants chose between certain gains $v s$ gambles to earn greater gains or no gains (inducing risk-aversion), or between certain losses vs gambles to suffer no loss or suffer still larger losses (inducing risk-seeking decisions) - the so-called 'reflection effect' (Schneider and Lopes, 1986). On these trials, there was no indication that THC attenuated the natural tendency to choose the risk-averse option in the former case and the risk-seeking option in the latter case compared to placebo. Therefore, while THC may interfere with motivational aspects of risky choice, and attention toward punishment cues under high-risk situations, there was little evidence that, in extremis, non-normative features of decisionmaking were disturbed.

Finally, the present findings have some clinical significance in the light of recent studies examining neuropsychological deficits, including decision-making, associated with chronic substance misuse (Rogers et al, 1999a). Whitlow et al (2004) have reported that heavy marijuana users ( 25 uses out of 30 days for 5 years) showed impaired decision-making on a card-playing task compared to light users (1-50 life time uses and no uses within the last year), perhaps reflecting the cumulative effects of previous drug use or pre-existing behavioral differences. Our results demonstrate that acute doses of THC also disrupt risky choice as measured by the present task paradigm. We have also examined performance of the same decision-making task by samples of current users of 3,4-methylenedioxymethamphetamine (MDMA), drug naive volunteers with no history of drug use at all, and polydrug users with a history of cannabis use equivalent to that of the MDMA users. While the MDMA users showed reduced attention towards gains and losses during risky choice compared to that of the other two samples, there was no difference between the decision-making of the polydrug (and cannabis) users and the nondrug using controls, suggesting that continued cannabis use does not impair risky choice as measured by this task (Morgan et al, 2006). Further work with decisionmaking tasks involving separable cognitive and affective components might clarify the connection between chronic cannabis use and risky choice.

\section{ACKNOWLEDGEMENTS}

This research was funded by an independent grant from GW Pharmaceuticals Ltd. to Robert D Rogers. Zubin Bhagwagar was funded by a MRC Clinical Training Fellowship.

\section{REFERENCES}

Ameri A (1999). The effects of cannabinoids on the brain. Prog Neurobiol 58: 315-348.

Baron J (2000). Thinking and Deciding, 3rd edn. Cambridge University Press: UK.

Bechara A, Damasio H (2002). Decision-making and addiction (part 1): impaired activation of somatic states in substance dependent individuals when pondering decisions with negative future consequences. Neuropsychologia 40: 1675-1689.

Bechara A, Dolan S, Denburg N, Hindes A, Anderson SW, Nathan PE (2001). Decision-making deficits linked to dysfunctional 
ventromedial prefrontal cortex, revealed in alcohol and stimulant abusers. Neuropsychologia 39: 376-389.

Bechara A, Tranel D, Damasio H, Damasio AR (1996). Failure to respond automatically to anticipated future outcomes following damage to prefrontal cortex. Cerebral Cortex 6: 215-225.

Bond A, Lader M (1974). The use of anologue scales in rating subjective feelings. Br J Med Psychol 47: 211-218.

Brady KT, Balster RL (1980). The effects of delta 9-tetrahydrocannabinol alone and in combination with cannabidiol on fixed interval performance in rhesus monkeys. Psychopharmacology 72: $21-26$.

Curran HV, Brignell C, Fletcher S, Middleton P, Henry J (2002). Cognitive and subjective dose-response effects of acute doses of oral $\Delta$-9-tetrahydrocannabinol (THC) in infrequent cannabis users. Psychopharmacology 164: 61-70.

Damasio AR (1994). Descartes Error. Grossman/Pullman: New York.

Dayan P, Balleine BW (2002). Reward, motivation, and reinforcement learning. Neuron 36: 285-298.

Deakin JB, Aitken MR, Dowson JH, Robbins TW, Sahakian BJ (2004). Diazepam produces disinhibitory cognitive effects in male volunteers. Psychopharmacology 173: 88-97.

First MB, Spitzer RL, Gibbon M, Williams JBW (1996). Structured Clinical Interview for DSM-IV Axis I Disorders. New York State Psychiatric Institute: New York.

Foltin RW, Fischman MW, Brady JV, Kelly TH, Bernstein DJ, Nellis MJ (1989). Motivational effects of smoked marijuana: behavioral contingencies and high probability recreational activities. Pharmacol Biochem Behav 34: 871-877.

Gardner EL, Lowinson JH (1991). Marijuana's interaction with brain reward systems: update 1991. Pharmacol Biochem Behav 40: 571-580.

Gardner EL, Paredes W, Smith D, Donner A, Milling C, Cohen D et al (1988). Facilitation of brain stimulation reward by delta 9-tetrahydrocannabinol. Psychopharmacology 96: 142-144.

Gardner EL, Vorel SR (1998). Cannabinoid transmission and reward-related events. Neurobiol Dis 5: 502-533.

Gevins A, Smith ME, McEvoy LK (2002). Tracking and cognitive pharmacodynamics of psychoactive substances with combinations of behavioral and neurophysiological measures. Neuropsychopharmacology 26: 27-39.

Goldstein WM, Hogarth RM (1997). Research on Judgment and Decision-Making. Currents, Connections and Controversies. Cambridge University Press: Cambridge, UK.

Guy GW, Robson PJ (2003). A Phase I, double-blind, three-way crossover study to assess the pharmacokinetic profile of cannabis based medicine extract (CBME) administered sublingually in variant cannabinoid ratios in normal healthy volunteers (GWPK0215). J Cannabis Therapeut 3: 121-152.

Haertzen CH, Hickey JE (1987). Addiction research center inventory (ARCI): measurement of euphoria and other drug effects. In: Bozarth MA (ed). Methods of Assessing the Reinforcing Properties of Abused Drugs. Springer-Verlag: New York. pp 489-524.

Haney M, Comer SD, Ward AS, Foltin RW, Fischman MW (1997). Factors influencing marijuana self-administration by humans. Behav Pharmacol 8: 101-112.

Hansteen RW, Miller RD, Lonero L, Reid LD, Jones B (1976). Effects of cannabis and alcohol on automobile driving and psychomotor tracking. Ann NY Acad Sci 282: 254-256.

Herkenham M, Lynn AB, Little MD, Johnson MR, Melvin LS, deCosta BR et al (1990). Cannabinoid receptor localization in brain. Proc Natl Acad Sci 87: 1932-1936.

Howell DC (1987). Statistical Methods for Psychology, 2nd edn. Duxbury Press: Boston. p 11.

Iversen LL (2000). The Science of Marijuana. Oxford University Press: Oxford.
Janowsky DS, Meacham MP, Blaine JD, Schoor M, Bozzetti LP (1976). Marijuana effects on simulated flying ability. Am J Psychiatry 133: 384-388.

Kagel JH, Battalio RC, Milus CG (1980). Marijuna and work performance: results from an experiment. J Human Reform 15: 373-395.

Kahneman D, Tversky A (1979). Prospect theory: an analysis of decision-making. Econometrica 47: 263-291.

Kahneman D, Tversky A (2000). Choices, Values and Frames. Cambridge University Press: Cambridge.

Kamien JB, Bickel WK, Higgins ST, Hughes JR (1994). The effects of $\Delta^{9}$-tetrahydrocannabinol on repeated acquisition and performance of response sequences and on self-report in humans. Behav Pharmacol 5: 71-78.

Kelly TH, Foltin RW, Fischman MW (1993). Effects of smoked marijuana on heart rate, drug ratings, and task performance by humans. Behav Pharmacol 4: 167-178.

Kirk JM, de Wit H (1999). Responses to oral $\Delta 9$-tetrahydrocannabinol in frequent and infrequent marijuana users. Pharmacol Biochem Behav 63: 137-142.

Kirk JM, Doty P, de Wit H (1998). Effects of expectancies on subjective responses to oral $\Delta 9$-tetrahydrocannabinol. Pharmacol Biochem Behav 59: 287-293.

Lane SD, Cherek DR (2002). Marijuana effects on sensitivity to reinforcement in humans. Neuropsychopharmacology 26: 520-529.

Lane SD, Cherek DR, Tcheremissine OV, Lieving LM, Pietras CJ (2005b). Acute effects of marijuana effects on human risk taking. Neuropsychopharmacology 30: 800-809.

Lane SD, Tcheremissine OV, Lieving LM, Nouvion SO, Cherek DR (2005a). Acute effects of alprazolam on risky decision making in humans. Psychopharmacology 181: 364-373.

Liguori A, Gatto CP, Robinson JH (1998). Effects of marijuana on equilibrium, psychomotor performance, and simulated driving. Behav Pharmacol 9: 599-609.

Luciana M, Depue RA, Arbisi P, Leon A (1992). Facilitation of working memory in humans by a D2 dopamine receptor agonist. J Cognitive Neurosci 4: 58-68.

Makela P, Wakeley J, Gijsman H, Robson PJ, Bhagwagar Z, Rogers RD (2006). Low doses of D-9 tetrahydrocannabinol (THC) have divergent effects on short-term spatial memory in young, healthy adults. Neurosychopharmacology 31: 462-470.

Mathew RJ, Wilson WH, Turkington TG, Hawk TC, Coleman RE, DeGrado TR et al (2002). Time course of tetrahydrocannabinolinduced changes in regional cerebral blood flow measured with positron emission tomography. Psychiatr Res 116: 173-185.

Mehta MA, Goodyer IM, Sahakian BJ (2004). Methylphenidate improves working memory and set-shifting in $\mathrm{AD} / \mathrm{HD}$ : relationships to baseline memory capacity. J Child Psychol Psychiatr 45: 293-305.

Miles CG, Congreve GR, Gibbins RJ, Marshman J, Devenyi P, Hicks RC (1974). An experimental study of the effects of daily cannabis smoking on behaviour patterns. Acta Pharmacol Toxicol 34(Suppl 1): 1-44.

Miller LL, McFarland DJ, Cornett TL, Brightwell DR, Wilker A (1977). Marijuana: effects on free recall and subjective organization of pictures and words. Psychopharmacology 55: 257-262.

Morgan MJ, Impallomeni LC, Pirona A, Rogers RD (2006). Elevated impulsivity and impaired decision-making in abstinent ecstasy (MDMA) users compared to polydrug and drug-naïve controls. Neuropsychopharmacology 31: 1562-1573.

Nelson HE (1982). National Adult Reading Test Manual. NFERNelson: Windsor, Berks.

O'Leary DS, Block RI, Koeppel JA, Flaum M, Schultz SK, Andreasen NC (2002). Effects of smoking marijuana on brain perfusion and cognition. Neuropsychopharmacology 26: 802-816. Pihl RO, Sigal H (1978). Motivation levels and the marihuana high. J Abnormal Psychol 87: 210-222. 
Rahman S, Sahakian BJ, Cardinal RN, Rogers RD, Robbins TW (2001). Decision-making cognition in neuropsychiatric disorders. Decision making and neuropsychiatry. Trends Cognitive Sci 5: 271-277.

Ramaekers JG, Berghaus G, van-Laar M, Drummer OH (2004). Dose related risk of motor vehicle crashes after cannabis use. Drug Alcohol Dependence 73: 109-119.

Ramaekers JG, Robbe HW, O'Hanlon JF (2000). Dose-related risk of motor vehicle crashes after cannabis use. Drug Alcohol Dependence 73: 109-119.

Robbe HWJ (1994). Influence of Marijuana on Driving. Institute of Human Psychopharmacology. University of Limburg: Maastricht.

Rogers RD, Everitt BJ, Baldacchino A, Blackmore AJ, Swainson R, London $M$ et al (1999a). Dissociating deficits in the decisionmaking cognition of chronic amphetamine abusers, opiate abusers, patients with focal damage to prefrontal cortex, and tryptophan-depleted normal volunteers: evidence for monoaminergic mechanisms. Neuropsychopharmacology 20: 322-329.

Rogers RD, Lancaster M, Wakeley J, Bhagwagar Z (2004). The effects of beta-adrenoceptor blockade on components of human decision-making. Psychopharmacology 172: 157-164.

Rogers RD, Owen AM, Middleton HC, Pickard J, Robbins TW (1999b). Decision-making in humans activates multiple sites within orbital prefrontal cortex: a PET study. J Neurosc 20: 9029-9038.

Rogers RD, Tunbridge EM, Bhagwagar Z, Drevets WC, Sahakian BJ, Carter CS (2003). Typtophan depletion alters the decisionmaking of healthy volunteers through altered processing of reward cues. Neuropsycholpharmacology 1: 1-10.
Scarna A, McTavish SFB, Cowen PJ, Goodwin GM, Rogers RD (2005). The effects of a branched chain amino acid mixture supplemented with tryptophan on biochemical indices of neurotransmitter function and decision-making. Psychopharmacology 179: 761-768.

Schneider SL, Lopes LL (1986). Reflection in preference under risk: who and when may suggest why. J Exp Psychol: Human Percept Performance 12: 535-548.

Schulze GE, McMillan DE, Bailey JR, Scallet AC, Ali SF, Slikker WJ et al (1989). Acute effects of marijuana smoke on complex operant behaviour in rhesus monkeys. Life Sci 45: 465-475.

Tanda G, Goldberg SR (2003). Cannabinoids: reward, dependence, and underlying neurochemical mechanisms - a review of recent preclinical data. Psychopharmacologia 169: 115-134.

Tanda G, Pontieri FE, DiChiara G (1997). Cannabinoid and heroin activation of mesolimbic dopamine transmission by a common mul opioid receptor mechanism. Science 276: 2048-2050.

Volkow ND, Gillespie H, Mullani N, Tancredi L, Grant C, Valentine A (1996). Brain glucose metabolism in chronic marijuana users at baseline and during marijuana intoxication. Psychiatry Res 67: 28-29.

von Winterfeldt D, Edwards W (1986). Decision Analysis and Behavioral Research. CUP: Cambridge.

Whitlow CT, Liguori A, Brooke Livengood L, Hart SL, MussatWhitlow BJ, Lambborn CM et al (2004). Long-term heavy marijuana use make costly decisions on a gambling task. Drug Alcohol Dependence 76: 107-111. 